\title{
Statistical Analyses of Electron Nanodiffraction Patterns to Examine Order and Structural Variability in Amorphous Materials
}

\author{
A. C. Y. Liu ${ }^{1,2}$, E. D. Bojesen ${ }^{1}$, P. Harrowell ${ }^{3}$ and T. C. Petersen ${ }^{2}$ \\ 1. Monash Centre for Electron Microscopy, Monash University, Clayton, Australia. \\ 2. School of Physics and Astronomy, Monash University, Clayton, Australia. \\ 3. School of Chemistry, University of Sydney, Camperdown, Australia.
}

The information contained in an ensemble of scanning electron nanodiffraction (END) patterns from amorphous materials is extremely rich and yet notoriously hard to interpret. By focussing the electron probe, the lateral size of the diffracting volume can be restricted to that of the "regions of correlated structure" (RCS) [1] and the otherwise isotropic diffuse amorphous rings in the diffraction pattern break up into areas of diffracted intensity that are related to the local structure. In reality, the diffracting volume has a finite thickness, spanning some tens of RCS. Atom positions in different RCS dispersed throughout the thickness may be uncorrelated, but diffraction from these pairs still contributes to the total diffracted intensity. This fact, and the complication of dynamical diffraction, means that, in general, the features in diffraction patterns from amorphous materials cannot be interpreted naively on the basis of a particular RCS [2,3]. Despite these difficulties, END patterns have been used to fingerprint local structure at the length scale of nearest-neighbour clusters [4,5] and map these subtle symmetries to [4] access any extended range order.

The correlations and symmetries found in these END patterns are weak, and interpretation has relied upon significant prior knowledge from related equilibrium crystal phases and atomistic modeling [4,5]. In this contribution we present more "order agnostic" approaches to decomposing these END data sets using a range of statistical analyses. In an isotropic material, such as an amorphous material, the local RCS can take any orientation with respect to the electron beam. Thus, one way to reduce the dimensionality of the data set is to remove the azimuthal dependence of the scattering angle by calculating the set of Fourier coefficients of the angular symmetries [4,5]. These Fourier coefficients can be compared to or decomposed into calculated fingerprints from ideal nearest-neighbour clusters [5].

Another approach is to calculate, without prior assumptions of structure, raw statistical measurements from this reduced data set of Fourier coefficients. For example, the covariance matrix can be calculated to investigate how different angular symmetries vary with respect to each other (Figure 1 (a)). The Pearson correlation coefficient can be mapped (Figure 1 (b)) or calculated from the whole ensemble of END patterns (Figure 1 (c)) to examine variability in the local structure from volume to volume. A Principal Component Analysis (Figure 1 (d)) can reveal the number of distinct orthogonal components required to encompass the data set and to identify the features that best describe the variance in the data. These measures can be compared for different specimens, providing more clues about the nature of local RCS and the variability and distribution of RCS. In some cases these measurements could provide an efficient and direct link to the underlying physics of how these materials form and behave, without requiring precise identification of local structure geometry. Indeed, these statistical correlative methods can accommodate structurally diverse systems for which the multiplicity of favoured local structures may be more important than the configuration of such clusters $[6,7][8]$. 
References:

[1] J M Cowley, Ultramicroscopy 90 (2002), p. 197.

[2] A C Y Liu et al, J Stat Mech (2016), p. 054046.

[3] A C Y Liu et al, Acta Cryst. A 71 (2015), p. 473.

[4] A C Y Liu et al, Phys Rev Lett 110 (2013), p. 205505.

[5] A C Y Liu et al, Phys Rev Lett 116 (2016), p. 205501.

[6] P Ronceray and P Harrowell, J Stat Mech, (2016), p. 084002.

[7] P Ronceray and P Harrowell, Soft Matter 11 (2015), p. 3322.

[8] The authors acknowledge use of the facilities at the Monash Centre for Electron Microscopy (FEI $\operatorname{Titan}^{3} 80-300$ FEGTEM funded by ARC LE0454166). Espen D Bojesen would like to thank VILLUM FONDEN for the funding via the research grant (VKR023371). We thank Joanne Etheridge, Laure Bourgeois and Matthew Weyland for many stimulating discussions.

Figure 1. Statistical measurements from a set of END patterns from a $\mathrm{Zr}_{36} \mathrm{Cu}_{64}$ metallic glass.

(a) Covariance matrix showing how different symmetries vary with respect to each other. (b) Map of the Pearson correlation coefficient showing how similar the Fourier coefficients of the central pixel are to others in the scanned array. (c) Correlation matrix of Fourier coefficients of all 900 END patterns from a scanned array. (d) Scree plot from a PCA analysis of Fourier coefficients of 900 END patterns.
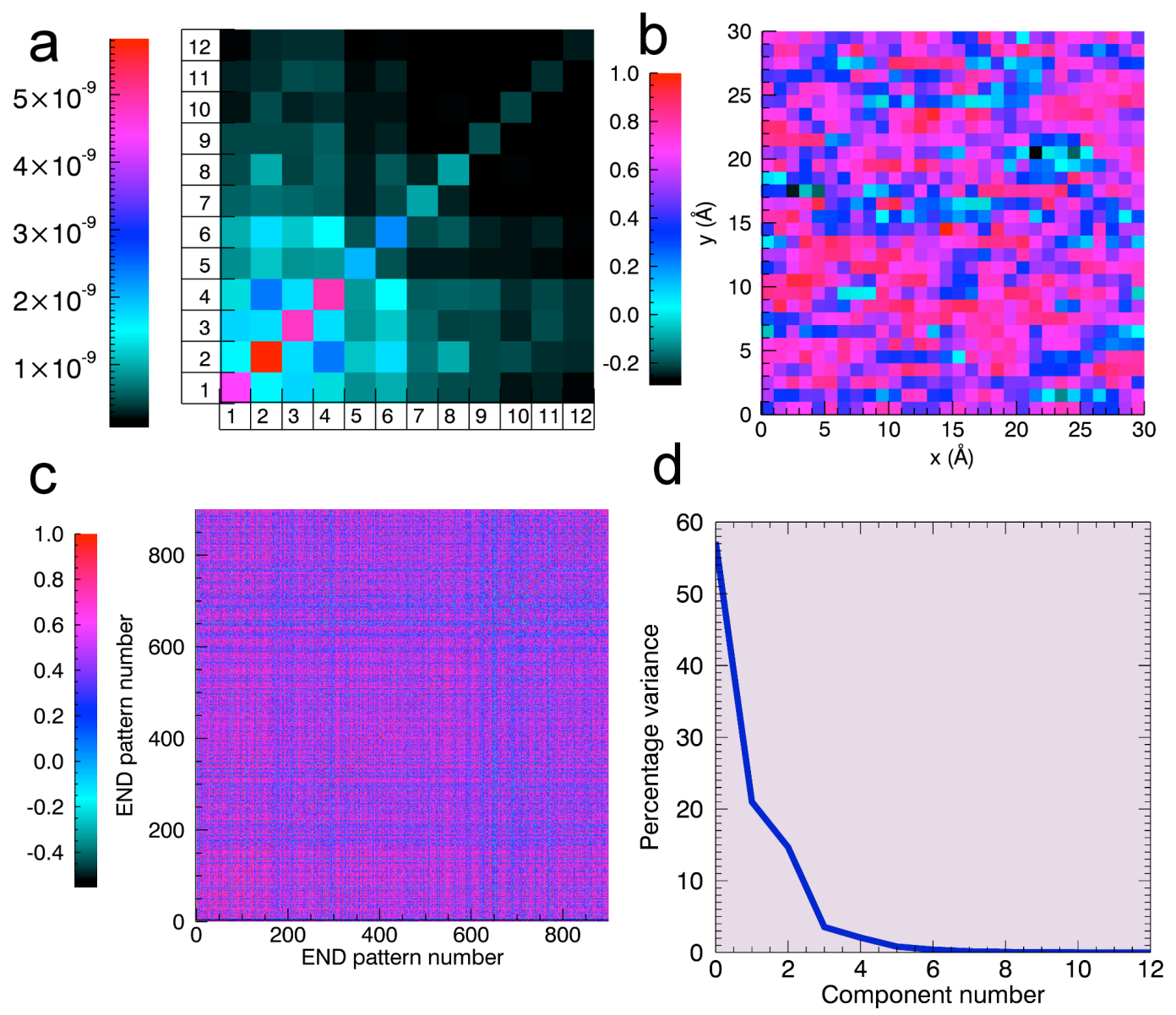